\title{
Random complex zeroes, I. Asymptotic normality
}

\author{
Mikhail Sodin* and Boris Tsirelson
}

\begin{abstract}
We consider three models (elliptic, flat and hyperbolic) of Gaussian random analytic functions distinguished by invariance of their zeroes distribution. Asymptotic normality is proven for smooth functionals (linear statistics) of the set of zeroes.
\end{abstract}

\section{Introduction and the main result}

Zeroes of random polynomials and other analytic functions were studied by mathematicians and physicists under various assumptions on random coefficients. One class of models introduced not long ago by Bogomolny, Bohigas and Leboeuf [5, 6], Kostlan [16], and Shub and Smale [23] has a remarkably unique unitary invariance:

"... indeed it has no true freedom at all. It is (statistically) unique in the same sense as 'the Poisson process', or 'the thermal (black body) electromagnetic field' are unique..."

Hannay [13, p. L755]

Following Hannay [12, we use the term 'chaotic analytic zero points' (CAZP, for short). We consider here three CAZP models: the elliptic CAZP, the flat CAZP, and the hyperbolic CAZP called by Leboeuf [17, p. 654] $S U(2)$, $W_{1}$, and $S U(1,1)$ in accordance with the symmetry group of the model. These models may be described analytically or geometrically. The analytical description is short and elementary: CAZP is the (random) set of zeroes of such a (random) analytic function $\psi$,

$$
\psi(z)=\sum_{k=0}^{L} \zeta_{k} \sqrt{\frac{L(L-1) \ldots(L-k+1)}{k !}} z^{k} \quad(\text { elliptic, } L=1,2, \ldots),
$$

\footnotetext{
${ }^{*}$ Supported by the Israel Science Foundation of the Israel Academy of Sciences and Humanities
} 


$$
\begin{array}{ll}
\psi(z)=\sum_{k=0}^{\infty} \zeta_{k} \sqrt{\frac{L^{k}}{k !}} z^{k} & (\text { flat }, L>0), \\
\psi(z)=\sum_{k=0}^{\infty} \zeta_{k} \sqrt{\frac{L(L+1) \ldots(L+k-1)}{k !}} z^{k} & \text { (hyperbolic, } L>0),
\end{array}
$$

where $\zeta_{0}, \zeta_{1}, \ldots$ are independent standard complex Gaussian (random) variables; that is, the distribution $\mathcal{N}_{\mathbb{C}}(0,1)$ of each $\zeta_{k}$ has the density $\pi^{-1} \exp \left(-|z|^{2}\right)$ with respect to the Lebesgue measure $m$ on $\mathbb{C}, d m(z)=(d \operatorname{Re} z)(d \operatorname{Im} z)$. For the elliptic CAZP one could assume equivalently that $\left(\zeta_{0}, \ldots, \zeta_{L}\right)$ is uniformly distributed on the sphere $\left|\zeta_{0}\right|^{2}+\cdots+\left|\zeta_{L}\right|^{2}=$ const (which changes the function $\psi$ without changing its zeroes). The analytic function (0.1) is a polynomial of degree $L$, the function (0.2) with probability one is an entire function (indeed, $\limsup \left|\zeta_{k}\right|^{1 / k}=1$ a.s.), and the function (0.3) with probability one is analytic in the unit disk.

Why just these coefficients? Because of symmetry of the models revealed by a geometric description, given in Sect. 1; readers not bothered by this question may skip that section.

We introduce unified notations: $\mathcal{M}$ for the domain of the random function, and $G$ for the symmetry group.

\begin{tabular}{|c|c|c|c|}
\hline & $\mathcal{M}$ (domain) & $\begin{array}{c}\text { invariant } \\
\text { metric }\end{array}$ & $G$ (symmetries) \\
\hline Elliptic & $\mathbb{C} \cup\{\infty\}$, that is, $\mathbb{S}^{2}$ & $\frac{|d z|}{1+|z|^{2}}$ & $z \mapsto \frac{a z+b}{-\bar{b} z+\bar{a}},|a|^{2}+|b|^{2}=1$ \\
Flat & $\mathbb{C}$ & $|d z|$ & $z \mapsto a z+b,|a|=1$ \\
Hyperbolic & $\mathbb{D}=\{z \in \mathbb{C}:|z|<1\}$ & $\frac{|d z|}{1-|z|^{2}}$ & $z \mapsto \frac{a z+b}{\bar{b} z+\bar{a}},|a|^{2}-|b|^{2}=1$ \\
\hline
\end{tabular}

A symmetry $g \in G$ transforms the random function $\psi$ into another random function $z \mapsto \psi(g(z))$ whose distribution depends on $g$. However, for every $g \in G$ there exists a complex-valued function $u_{g}$ on $\mathcal{M},\left|u_{g}(z)\right|=1$, such that two random functions

$$
z \mapsto \frac{\psi(g(z))}{\|\psi(g(z))\|} \quad \text { and } \quad z \mapsto u_{g}(z) \frac{\psi(z)}{\|\psi(z)\|}
$$

are identically distributed. Here

$$
\|\psi(z)\|=\left(\mathbb{E}|\psi(z)|^{2}\right)^{1 / 2}= \begin{cases}\left(1+|z|^{2}\right)^{L / 2} & \text { (elliptic) } \\ \exp \left(L|z|^{2} / 2\right) & \text { (flat), } \\ \left(1-|z|^{2}\right)^{-L / 2} & \text { (hyperbolic) } .\end{cases}
$$


For example, a shift $g(z)=z+b$ (for the flat case); here $u_{g}(z)=\exp (i L \operatorname{Im}(z \bar{b}))$. The symmetry ensures that the distribution of the (random) set of zeroes of $\psi$ is invariant under $G$. For details, see Sect. 1.

In each of the three cases we have a parameter $L$ which is assumed to be large. Increasing $L$, we increase accordingly the mean number of random zeroes per unit area of $\mathcal{M}$.

In the flat case, there is another interpretation: introducing $L$, we just make a homothety of the plane with coefficient $\sqrt{L}$. This makes some computations simpler.

In the other two models, changing the value of the parameter $L$, we can change the properties of the process. We mention a recent discovery of Peres and Virág [21] that in the special case $L=1$ of the hyperbolic model, the point process is a determinantal one [26]. In this case, Peres and Virág found a simple expression for $n$-point correlation functions and explicitly computed the distribution of random zeroes in discs in $\mathbb{D}$.

Below, the asymptotic normality is stated for smooth functionals of the random set of zeroes, namely, for the random variables (often called the linear statistics of zeroes)

$$
Z_{L}(h)=\sum_{z: \psi_{L}(z)=0} h(z)=\int_{\mathcal{M}} h d n_{\psi_{L}}
$$

where $h$ is a smooth test function, and $n_{\psi}$ is the counting measure on the set of zeroes. In the elliptic case, $n_{\psi_{L}}\left(\mathbb{S}^{2}\right)=L$; in the other two cases $n_{\psi}$ is infinite but locally finite. The choice of the test-functions depends on the model: in the elliptic case $h$ is a real-valued $C^{2}$-function on the Riemann sphere, in the flat case $h$ is a real-valued $C^{2}$-function with a compact support in the plane, and in the hyperbolic case $h$ is a $C^{2}$-function with a compact support in the unit disk. Multiple zeroes may be ignored, as well as a possible zero at $\infty$ in the elliptic case, since it happens with zero probability.

ExpeCtation of the Linear statistics. For the elliptic model, invariance itself gives us the expectation of the random variable $Z_{L}(h)$; in the other two cases it defines the expectation up to a numerical coefficient which can be easily found using the Edelman-Kostlan formula $\mathbb{E} n_{\psi}=(2 \pi)^{-1} \Delta \log \|\psi\| d m$ (see [9], 24]):

$$
\mathbb{E} Z_{L}(h)=L \frac{1}{\pi} \int_{\mathcal{M}} h d m^{*}
$$

where

$$
d m^{*}(z)= \begin{cases}d m(z) & \text { (flat) } \\ \left(1+|z|^{2}\right)^{-2} d m(z) & \text { (elliptic) } \\ \left(1-|z|^{2}\right)^{-2} d m(z) & \text { (hyperbolic) }\end{cases}
$$


is the invariant measure on $\mathcal{M}$.

Fluctuations of the linear statistics. These are computed in Section 2. We get

$$
\operatorname{Var} Z_{L}(h)=\frac{\kappa}{L}\left\|\Delta^{*} h\right\|_{L^{2}\left(m^{*}\right)}^{2}+o\left(L^{-1}\right), \quad L \rightarrow \infty
$$

where $\kappa$ is a numerical constant (the same for each of the three cases), and $\Delta^{*}$ is the invariant Laplacian on $\mathcal{M}$ :

$$
\Delta^{*} h(z)= \begin{cases}\Delta h(z) & \text { (flat) } \\ \left(1+|z|^{2}\right)^{2} \Delta h(z) & (\text { elliptic) } \\ \left(1-|z|^{2}\right)^{2} \Delta h(z) & \text { (hyperbolic) }\end{cases}
$$

This shows a decay of the fluctuations of the linear statistics. ${ }^{1}$ Note that in the flat case formula (0.6) was found in a paper by Forrester and Honner [10], in which they also conjectured the asymptotics in the elliptic case and checked it numerically. The estimate $\operatorname{Var} Z_{L}(h)=O(1), L \rightarrow \infty$, was obtained by Shiffman and Zelditch in 22$]$.

Main Theorem. In each of the three cases, the random variables

$$
\frac{\sqrt{L}}{\sqrt{\kappa}\left\|\Delta^{*} h\right\|_{L^{2}\left(m_{*}\right)}}\left(Z_{L}(h)-L \frac{1}{\pi} \int_{\mathcal{M}} h d m^{*}\right)
$$

converge in distribution to $\mathcal{N}(0,1)$ for $L \rightarrow \infty$.

The proof of Main Theorem is based on the asymptotic normality theorem for non-linear functionals of Gaussian processes. Results of this type are known; usually, their proofs rely on the classical method of moments combined with the diagram technique (see Breuer and Major [7, and the references therein). In Section 2 we shall prove another result in that spirit using a similar strategy. In Section 3, we deduce Main Theorem from this result and prove the asymptotics (0.6).

For real random polynomials $P_{N}(x)=\sum_{k=0}^{N} \xi_{k} x^{k}$ where $\xi_{k}$ are independent identically distributed (real-valued) random variables such that $\mathbb{E} \xi_{k}=0$, $\mathbb{P}\left(\xi_{k}=0\right)=0$, and $\mathbb{E}\left|\xi_{k}\right|^{2+\epsilon}<\infty$, Maslova [18], [19] evaluated the variance

\footnotetext{
${ }^{1}$ The situation changes if we allow non-smooth test functions $h$. In particular, for the flat model, the variance of the number of random zeroes in a smooth domain $\Lambda$ is asymptotic to $\sqrt{L}$ times the perimeter of $\Lambda$, see [10]. The difference between these two types of behavior reflects high frequency oscillations of random zeroes which are not taken into account when the test function is smooth.
} 
of the number of real zeroes of $P_{N}$, and proved the corresponding version of asymptotic normality. Her methods are quite different from ours. Probably, Main Theorem persists for more general models like zeroes of random holomorphic sections of high powers of Hermitian line bundles over Kähler manifolds extensively studied by Bleher, Shiffman and Zelditch [22, 2, 3].

Three TOY MODELS FOR THE FLAT CAZP. It is instructive to compare the flat CAZP with simpler ('toy') models of random point processes in the plane, especially, random perturbations of a lattice. The first toy model: each point of the lattice $\sqrt{\frac{\pi}{2}} \mathbb{Z}^{2}=\left\{\sqrt{\frac{\pi}{2}}(k+l i): k, l \in \mathbb{Z}\right\}$ is deleted at random, independently of others, with probability $1 / 2$; the remaining points are a random set. The corresponding smooth linear statistics $Z_{L}^{(1)}(h)=\int h(z / \sqrt{L}) d n^{(1)}(z)$ (where $n^{(1)}$ is the counting measure on the random set) is asymptotically normal with parameters $\mathbb{E} Z_{L}^{(1)}(h)=L \pi^{-1} \int h d m$ and $\operatorname{Var} Z_{L}^{(1)}(h)=\pi^{-1} \int h^{2}(z / \sqrt{L}) d m(z)=L \pi^{-1}\|h\|_{L^{2}(m)}^{2}$, which is quite different from (0.6): the variance grows, not decays; and $\|h\|$ appears, not $\|\Delta h\|$.

The second toy model: points of the lattice $\sqrt{\pi} \mathbb{Z}^{2}$ move independently, forming the random set $\left\{\sqrt{\pi}(k+l i)+c \eta_{k, l}: k, l \in \mathbb{Z}\right\}$ where $\eta_{k, l}$ are independent standard complex Gaussian random variables, and $c \in(0, \infty)$ is a parameter. The corresponding $Z_{L}^{(2)}(h)=\int h(z / \sqrt{L}) d n^{(2)}(z)$ is asymptotically normal with parameters $\mathbb{E} Z_{L}^{(2)}(h)=(1+o(1)) L \pi^{-1} \int h d m$ and $\operatorname{Var} Z_{L}^{(2)}(h)=\left(c^{2}+o(1)\right)(2 \pi)^{-1}\|\nabla h\|_{L^{2}(m)}^{2}$ (where $\nabla$ stands for gradient). This time, the variance does not grow, but still, it does not decay; and $\|\nabla h\|$ is not $\|\Delta h\|$.

The third toy model reaches asymptotic similarity to the flat CAZP, but is more complicated. Lattice points are initially aggregated into three-point clusters, and each cluster scatters in a special (equiangular and equidistant) way. Namely, we consider the random set

$$
\left\{\sqrt{3 \pi}(k+l i)+c e^{2 \pi i m / 3} \eta_{k, l}: k, l \in \mathbb{Z} ; m=0,1,2\right\},
$$

where $\eta_{k, l}$ and $c$ are as before. The corresponding $Z_{L}^{(3)}(h)=\int h(z / \sqrt{L}) d n^{(3)}(z)$ is asymptotically normal with parameters $\mathbb{E} Z_{L}^{(3)}(h)=(1+o(1)) L \pi^{-1} \int h d m$ and $\operatorname{Var} Z_{L}^{(3)}(h)=(1+o(1)) L^{-1} 3 c^{4}(16 \pi)^{-1}\|\Delta h\|_{L^{2}(m)}^{2}$. This time, we can mimic (0.6) by choosing $c=2(\pi \kappa / 3)^{1 / 4}$.

We come to a vague idea of two conservation laws for random zeroes: mass conservation and center-of-mass conservation. Mass is conserved in the second and third toy models; center-of-mass is conserved in the third toy model only. Both should be conserved in the flat CAZP, in some sense (to 
be understood). An attempt to understand the mass conservation is made in our next work [25]. The center-of-mass conservation remains unexplored.

About the three directions $\left(e^{2 \pi i m / 3}: m=0,1,2\right)$ : four or more directions may be used equally well, but two directions are not enough. Indeed, every quadratic form $Q: \mathbb{C} \rightarrow \mathbb{R}$ satisfies $\frac{1}{n} \sum_{m=0}^{n-1} Q\left(e^{2 \pi i m / n}\right)=\frac{1}{4} \Delta Q(0)$ provided that $n \geq 3$; however, $Q(1)+Q(-1)$ is not proportional to $\Delta Q(0)$.

Maybe, the failure of two-point clusters hints at a third conservation law.

Acknowledgment. We thank Leonid Pastur, Leonid Polterovich and Zeev Rudnik for useful discussions.

\section{Geometrical description of models}

By the geometrical description of CAZP we mean something like this:

The CAZP process on a complex 1-dimensional analytic manifold is the intersection of its isometric embedding into a projective space with a random hyperplane.

However, we do not formalize the description; we only explain, how it works in the three models considered. For related more advanced theories, see the works of Gromov [11, Sect. 3.3], Shub and Smale [23, and Bleher, Shiffman and Zelditch [4].

\section{$1.1 \quad$ Three homogeneous spaces}

Each model is based on a simply connected homogeneous space $\mathcal{M}$; it may be thought of as a real 2-dimensional manifold or a complex 1-dimensional analytic manifold. ${ }^{2}$ The former may be embedded into a Euclidean space, the latter - into the projective space $P\left(\mathbb{C}^{2}\right)$ of 1-dimensional subspaces of $\mathbb{C}^{2}$. The complex plane $\mathbb{C}$ is embedded into $P\left(\mathbb{C}^{2}\right)$ by $z \mapsto\{(u, u z): u \in \mathbb{C}\}$; in order to cover the whole $P\left(\mathbb{C}^{2}\right)$, one additional point is needed, $\infty \mapsto$ $\{(0, u): u \in \mathbb{C}\}$. The symmetry group $G$ in the real case is a subgroup of the group of motions of the Euclidean space. In the complex case, $G$ is a subgroup of the group of projective transformations of $P\left(\mathbb{C}^{2}\right)$. The latter is covered by the group $S L(2)$, and we may take $G \subset S L(2)$; such an action need not be effective (since $(-1)$ acts trivially), which is harmless.

\footnotetext{
${ }^{2}$ The choice of a complex structure on our real manifold does not introduce arbitrariness; there exist only two invariant complex structures, conjugate to each other, both leading to the same CAZP.
} 
ELLIPTIC MODEL. The real manifold is the sphere

$$
\mathbb{S}^{2}=\left\{\left(x_{0}, x_{1}, x_{2}\right): x_{0}^{2}+x_{1}^{2}+x_{2}^{2}=1\right\} \subset \mathbb{R}^{3},
$$

the symmetry group being $S O(3)$ (orientation-preserving rotations of $\mathbb{R}^{3}$ ). The complex manifold is the whole $P\left(\mathbb{C}^{2}\right)$, with $G=S U(2)$; or alternatively, $\mathbb{C} \cup\{\infty\}$ with transformations $z \mapsto(a z+b) /(c z+d),\left(\begin{array}{ll}a & b \\ c & d\end{array}\right) \in S U(2)$, preserving the spherical metric $|d z| /\left(1+|z|^{2}\right)$. The correspondence (well-known as the stereographic projection) is $z=\left(x_{1}+i x_{2}\right) /\left(1-x_{0}\right)$. See also [17, Sect. 2] and [20, Chap. 4].

FLAT MODEL. The real manifold is the plane $\mathbb{R}^{2}$ with the group of (orientationpreserving) motions, that is, shifts and rotations. The complex manifold is the complex plane $\mathbb{C}$ with transformations $z \mapsto e^{i \varphi}(z+u)$, preserving the Euclidean metric $|d z|$; or alternatively, $P\left(\mathbb{C}^{2}\right)$ minus a single point, with transformations preserving the point. The correspondence is just $z=x_{1}+i x_{2}$.

Hyperbolic MODEL. The real manifold is $\left\{\left(x_{0}, x_{1}, x_{2}\right): x_{0}=\sqrt{1+x_{1}^{2}+x_{2}^{2}}\right\}$ $\subset \mathbb{R}^{3}$, the upper sheet of a hyperboloid (in other words, a pseudosphere), the symmetry group being the connected component of $S O(2,1)$ (rotations of $\mathbb{R}^{3}$ that preserve the sheet and orientation). The complex manifold is the $\operatorname{disc} \mathbb{D}=\{z \in \mathbb{C}:|z|<1\}$, with transformations $z \mapsto(a z+b) /(\bar{b} z+\bar{a})$, $|a|^{2}-|b|^{2}=1$, preserving the hyperbolic metric $|d z| /\left(1-|z|^{2}\right)$; or alternatively, the corresponding part of $P\left(\mathbb{C}^{2}\right)$, with $G=S U(1,1)$ (isomorphic to $S L(2, \mathbb{R}))$. The correspondence is $z=\left(x_{1}+i x_{2}\right) /\left(1+x_{0}\right)$. See also [17, Sect. 2] and [20, Chap. 5].

\subsection{Enlarging symmetry}

The symmetry group $G$ acts transitively on $\mathcal{M}$. It acts also on discrete subsets of $\mathcal{M}$, but not transitively. A $G$-invariant random discrete subset of $\mathcal{M}$ is far from being unique in distribution.

The key ingredient of the construction is an embedding $\iota$ of $\mathcal{M}$ into a high-dimensional projective space $P\left(\mathbb{C}^{n}\right), n \in\{1,2, \ldots\} \cup\{\infty\}$, and use of the high-dimensional symmetry group $U(n)$ of rotations of $\mathbb{C}^{n}$. Of course, the image $\iota(\mathcal{M})$ is not $U(n)$-invariant. However, we use $U(n)$-invariance for determining a probability measure on hyperplanes. Choosing at random a hyperplane, we observe its intersection with $\iota(\mathcal{M})$. The case $n=\infty$ does involve some technicalities; we will return to the point later.

The embedding must be $(G, U(n))$-invariant in the following sense: for 
every $g \in G$ there exists $U \in U(n)$ such that the diagram

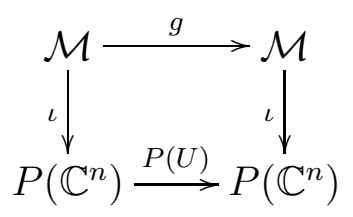

is commutative. Surprisingly, such an embedding is unique (up to a rotation)! Uniqueness of $\iota$ leads to a model, (statistically) unique in the same sense as the Poisson process (recall the quote from Hannay in the Introduction).

Now we switch from a heuristic to rigorous style.

Let $H$ be a Hilbert space over $\mathbb{C}$, either finite-dimensional, or infinitedimensional and separable. The projective space $P(H)$ is, by definition, the set of all one-dimensional subspaces of $H$. Each non-zero vector $x \in$ $H \backslash\{0\}$ spans such a subspace $P(x) \in P(H)$. A transformation $P(U)$ : $P(H) \rightarrow P(H)$ corresponds to every unitary operator $U \in U(H)$; namely, $P(U)(P(x))=P(U x)$. Note that $P\left(e^{i \varphi} U\right)=P(U)$. Two maps $\iota, \iota^{\prime}: \mathcal{M} \rightarrow$ $P(H)$ are called unitarily equivalent, if $\iota^{\prime}=P(U) \circ \iota$ for some $U \in U(H)$. A map $\iota: \mathcal{M} \rightarrow P(H)$ is called holomorphic, if locally (in a neighborhood of any point of $\mathcal{M})$ it is the composition of some holomorphic map $\mathcal{M} \rightarrow H \backslash\{0\}$ and the canonical projection $H \backslash\{0\} \rightarrow P(H), x \mapsto P(x)$.

The well-known Fubini-Study metric ${ }^{3}$ on $P(H)$,

$$
\operatorname{dist}(P(x), P(y))=\arccos \frac{|\langle x, y\rangle|}{\|x\|\|y\|},
$$

is $U(n)$-invariant. Given a one-to-one map $\iota: \mathcal{M} \rightarrow P(H)$, we get a metric on $\mathcal{M}$,

$$
\operatorname{dist}_{\iota}\left(z, z^{\prime}\right)=\operatorname{dist}\left(\iota(z), \iota\left(z^{\prime}\right)\right)
$$

assuming smoothness of $\iota$ and taking $z^{\prime}$ infinitesimally close to $z$ we get a tensor field $g_{\iota}$ on $\mathcal{M}$, the Riemannian metric induced by $\iota$. If $\iota$ and $\iota^{\prime}$ are unitarily equivalent, then they induce the same Riemannian metric (since $\operatorname{dist}_{\iota}=$ dist $\left._{\iota^{\prime}}\right)$.

Calabi's rigidity theorem. If two holomorphic embeddings ${ }^{4}$ of a complex manifold into $P(H)$ induce the same Riemannian metric on $\mathcal{M}$, then they are unitarily equivalent. ([8, Th. 9], see also [27, Sect. 4].)

\footnotetext{
${ }^{3}$ Or rather, the geodesic metric corresponding to the Fubini-Study metric tensor, up to a coefficient.

${ }^{4}$ 'Embedding' means one-to-one. The theorem holds also for immersions, but we need only embeddings.
} 
If a holomorphic embedding $\iota: \mathcal{M} \rightarrow P(H)$ is $(G, U(n))$-invariant (recall (1.11)), then it induces a $G$-invariant Riemannian metric on $\mathcal{M}$. However, such a metric is unique up to a coefficient (since $G$ can rotate $\mathcal{M}$ around any given point). It means that the coefficient is the only possible parameter of a holomorphic $(G, U(n))$-invariant embedding $\iota: \mathcal{M} \rightarrow P(H)$ (treated up to unitary equivalence).

Another implication of Calabi's theorem: if a holomorphic embedding $\iota: \mathcal{M} \rightarrow P(H)$ induces a $G$-invariant Riemannian metric on $\mathcal{M}$, then $\iota$ is $(G, U(n))$-invariant.

By the way, $(G, U(n))$-invariance leads to a projective representation of $G$ (that is, a homomorphism from $G$ to the factor group of $U(H)$ by its center), not necessarily a homomorphism $G \rightarrow U(H)$.

\subsection{Relation to the analytical description}

In order to finish the description, it remains to write down the corresponding embeddings $\iota$ in each of the three cases, to check invariance of induced metrics, and to explain the rotation invariant choice of the random hyperplane in the case when the dimension of $H$ is infinite.

All invariant metrics on $\mathcal{M}$ are proportional to the one mentoned in the Introduction. The only freedom left to us is to choose the numerical coefficient $\sqrt{L}$ of the invariant metric on $\mathcal{M}$.

ELLIPTIC CASE. For the sphere $\mathbb{S}^{2}$, the existence of such an embedding depends on the parameter $L$. Only for $L=1,2,3, \ldots$ the invariant metric on $\mathbb{S}^{2}$ is embeddable into $P(H) .{ }^{5}$ For such $L$, an embedding $\mathbb{S}^{2} \rightarrow P\left(\mathbb{C}^{L+1}\right)$ is well-known to physicists as the system of spin- $J$ coherent states $(J=$ $L / 2$ ), see [12, [20, Sect. 4.3], 27]. Sometimes, mathematicians call the wave function of a coherent state "the Szegö kernel" (see [3]). Treated up to rotations of $\mathbb{C}^{L+1}$, the map is unique. By rigidity, every such embedding uses only a finite-dimensional subspace of $H$.

The embedding $\mathbb{S}^{2} \rightarrow P\left(\mathbb{C}^{L+1}\right)$ may be described as the composition $\mathbb{S}^{2} \rightarrow \mathbb{C} \rightarrow \mathbb{C}^{L+1} \backslash\{0\} \rightarrow P\left(\mathbb{C}^{L+1}\right)$, where $\mathbb{S}^{2} \rightarrow \mathbb{C}$ is the stereographic projection mentioned in Sect. 1.1, $\mathbb{C}^{L+1} \backslash\{0\} \rightarrow P\left(\mathbb{C}^{L+1}\right)$ is the canonical projection $x \mapsto P(x)$, and $\tilde{\iota}: \mathbb{C} \rightarrow \mathbb{C}^{L+1} \backslash\{0\}$ is given by

$$
\tilde{\iota}(z)=\left(\sqrt{\left(\begin{array}{l}
L \\
0
\end{array}\right)}, \sqrt{\left(\begin{array}{c}
L \\
1
\end{array}\right)} z, \ldots, \sqrt{\left(\begin{array}{l}
L \\
L
\end{array}\right)} z^{L}\right),
$$

which evidently corresponds to (0.1).

\footnotetext{
${ }^{5}$ One can easily get this using the Edelman-Kostlan formula: if $L$ is non-integer, then we would get a non-integer answer for the average number of zeroes of our random function on $\mathbb{S}^{2}$.
} 
The induced metric is easy to calculate:

$$
\begin{gathered}
\left\langle\tilde{\iota}(z), \tilde{\iota}\left(z^{\prime}\right)\right\rangle=\sum_{k=0}^{L} \sqrt{\left(\begin{array}{l}
L \\
k
\end{array}\right)} z^{k} \sqrt{\left(\begin{array}{l}
L \\
k
\end{array}\right)} \bar{z}^{\prime k}=\left(1+z \bar{z}^{\prime}\right)^{L} ; \\
\operatorname{dist}_{\iota}\left(z, z^{\prime}\right)=\arccos \frac{\left|\left\langle\tilde{\iota}(z), \tilde{\iota}\left(z^{\prime}\right)\right\rangle\right|}{\|\tilde{\iota}(z)\|\left\|\tilde{\iota}\left(z^{\prime}\right)\right\|}=\arccos \left(\frac{\left|1+z \bar{z}^{\prime}\right|}{\sqrt{1+|z|^{2}} \sqrt{1+\left|z^{\prime}\right|^{2}}}\right)^{L} .
\end{gathered}
$$

$G$-invariance of the metric for every $L$ follows immediately from its $G$-invariance for $L=1$; however, for $L=1$ the embedding $\mathbb{C} \rightarrow P\left(\mathbb{C}^{L+1}\right)$, given by $z \mapsto\{(u, u z): u \in \mathbb{C}\}$, is already familiar to us (recall the beginning of Sect. 1.1). An explicit calculation gives for $\Delta z \rightarrow 0$

$$
\begin{aligned}
& \operatorname{dist}_{\iota}(z-\left.\frac{1}{2} \Delta z, z+\frac{1}{2} \Delta z\right) \\
&=\arccos \left(1-\frac{|\Delta z|^{2}}{\left(1+|z|^{2}+\frac{1}{4}|\Delta z|^{2}\right)^{2}-\operatorname{Re}^{2}(\bar{z} \Delta z)}\right)^{L / 2} \\
&=\arccos \left(1-\frac{L}{2} \frac{|\Delta z|^{2}}{\left(1+|z|^{2}\right)^{2}}(1+o(1))\right)=\sqrt{L} \frac{|\Delta z|}{1+|z|^{2}}(1+o(1)) .
\end{aligned}
$$

FLAT CASE. If the needed embedding $\iota: \mathbb{C} \rightarrow P(H)$ exists for $L=1$, then for every $L \in(0, \infty)$ the map $z \mapsto \iota\left(L^{1 / 2} z\right)$ fits; we restrict ourselves to $L=1$. The construction of $\iota$ is well-known to physicists as the usual system of coherent states (Schrödinger, von Neumann, Klauder, et al.), see [20, Chapter 1]. Its explicit form is given (similarly to the elliptic case) by the composition $\mathbb{C} \rightarrow H \backslash\{0\} \rightarrow P(H)$ where the first map $\tilde{\iota}: \mathbb{C} \rightarrow H \backslash\{0\}$ is given by

$$
\tilde{\iota}(z)=\left(1, z, \frac{z^{2}}{\sqrt{2 !}}, \frac{z^{3}}{\sqrt{3 !}}, \ldots\right) \in l^{2}=H,
$$

cf. (0.2).

We have

$$
\left\langle\tilde{\iota}(z), \tilde{\iota}\left(z^{\prime}\right)\right\rangle=\sum_{k=0}^{\infty} \frac{z^{k}}{\sqrt{k !}} \frac{\bar{z}^{\prime k}}{\sqrt{k !}}=\exp \left(z \bar{z}^{\prime}\right)
$$

$$
\begin{aligned}
& \operatorname{dist}_{\iota}\left(z, z^{\prime}\right)=\arccos \exp \left(\operatorname{Re}\left(z \bar{z}^{\prime}\right)-\frac{1}{2}|z|^{2}\right.\left.-\frac{1}{2}\left|z^{\prime}\right|^{2}\right) \\
&=\operatorname{arccosexp}\left(-\frac{1}{2}\left|z-z^{\prime}\right|^{2}\right) \\
& \operatorname{dist}_{\iota}(z, z+\Delta z)=\arccos \left(1-\frac{1}{2}|\Delta z|^{2}(1+o(1))\right)=|\Delta z|(1+o(1)) .
\end{aligned}
$$


HyperBoliC CASE. The corresponding system of coherent states is usually investigated in terms of unitary representations of the group $G=S U(1,1)$, see [20, Chap. 5], [17, Sect. 2], which leads to a special treatment of integer values of $L$. In our approach, projective representations appear irrespective of unitary representations, and $L$ runs over $(0, \infty)$.

As before, $\iota(z)=P(\tilde{\iota}(z))$, but now $z \in \mathbb{D}$, and $\tilde{\iota}: \mathbb{D} \rightarrow l^{2}$ is given by

$$
\tilde{\iota}(z)=\left(1, \sqrt{L} z, \sqrt{\frac{L(L+1)}{2 !}} z^{2}, \sqrt{\frac{L(L+1)(L+2)}{3 !}} z^{3}, \ldots\right),
$$

cf. (0.3). The corresponding embedding of the hyperbolic plane to $P(H)$ was known already to Bieberbach in 1932. We have

$$
\begin{gathered}
\left\langle\tilde{\iota}(z), \tilde{\iota}\left(z^{\prime}\right)\right\rangle=\sum_{k=0}^{\infty} \sqrt{\left(\begin{array}{c}
L+k \\
k
\end{array}\right)} z^{k} \sqrt{\left(\begin{array}{c}
L+k \\
k
\end{array}\right)} \bar{z}^{\prime k}=\left(1-z \bar{z}^{\prime}\right)^{-L} ; \\
\operatorname{dist}_{\iota}\left(z, z^{\prime}\right)=\arccos \left(\frac{\left|1-z \bar{z}^{\prime}\right|}{\sqrt{1-|z|^{2}} \sqrt{1-\left|z^{\prime}\right|^{2}}}\right)^{-L} ; \\
\operatorname{dist}_{\iota}\left(z-\frac{1}{2} \Delta z, z+\frac{1}{2} \Delta z\right) \\
=\arccos \left(1+\frac{|\Delta z|^{2}}{\left(1-|z|^{2}-\frac{1}{4}|\Delta z|^{2}\right)^{2}-\operatorname{Re}^{2}(\bar{z} \Delta z)}\right)^{-L / 2} \\
=\arccos \left(1-\frac{L}{2} \frac{|\Delta z|^{2}}{\left(1-|z|^{2}\right)^{2}}(1+o(1))\right)=\sqrt{L} \frac{|\Delta z|}{1-|z|^{2}}(1+o(1)) .
\end{gathered}
$$

\subsection{Random hyperplane in Hilbert space?}

In $\mathbb{C}^{n}$, the only $U(n)$-invariant distribution on hyperplanes, the uniform distribution, may be represented via the normal (to the hyperplane) vector $\left(\zeta_{1}, \ldots, \zeta_{n}\right)$ distributed uniformly on the sphere. The normal distribution for $\left(\zeta_{1}, \ldots, \zeta_{n}\right)$ can be used instead (as well as any spherically invariant distribution).

In a Hilbert space $H=l^{2}$ there is no rotation-invariant probability measure on hyperplanes (nor Lebesgue measure in $H$ ). Nevertheless, an infinite sequence of independent normal $\mathcal{N}_{\mathbb{C}}(0,1)$ random variables $\zeta_{k}$ can be used. Of course, it is not a random element of $H$, since the event $\sum\left|\zeta_{k}\right|^{2}<\infty$ is of probability 0 . However, for every $x=\left(c_{1}, c_{2}, \ldots\right) \in l^{2}$ the series $\sum c_{k} \bar{\zeta}_{k}$ converges a.s., and we may denote its sum by $\langle x, \zeta\rangle$. The 'bad' set of zero probability, on which the series does not converge, depends on $x$; the union 
over all $x \in H$ is not a set of zero probability. ${ }^{6}$ We cannot choose $\zeta$ at random and speak about 'the function $x \mapsto\langle x, \zeta\rangle$ on $H$ '.

What we really need, is the function $z \mapsto\langle\tilde{\iota}(z), \zeta\rangle$ for $z \in \mathcal{M}$, that is, the function $x \mapsto\langle x, \zeta\rangle$ for $x \in \tilde{\iota}(\mathcal{M})$ only. The corresponding series converges a.s. for all these $x$ simultaneously. Thus, 'the random hyperplane of $H$ ' is ill-defined, but still, its intersection with $\tilde{\iota}(\mathcal{M})$ is well-defined.

It remains to explain unitary invariance of the construction described above. Random variables $\langle x, \zeta\rangle$ can be used simultaneously for a countable set of points $x$. In particular, for every orthonormal basis $\left(e_{1}, e_{2}, \ldots\right)$ of $H$, the sequence of random variables $\left\langle e_{k}, \zeta\right\rangle$ is well-defined. Some reflection shows that the joint distribution of these $\left\langle e_{k}, \zeta\right\rangle$ does not differ from that of $\zeta_{k}$. It follows easily that our construction can start with an arbitrary basis, resulting in the same distribution of the random function $z \mapsto\langle\tilde{\iota}(z), \zeta\rangle$. See also [14, Sect. 1.3].

\section{Asymptotic normality for non-linear func- tionals of Gaussian processes}

\subsection{The result}

Let $T$ be a measure space endowed with a finite positive measure $\mu, \mu(T)=1$. A complex-valued Gaussian process on $T$ may be defined as

$$
w(\omega, t)=\sum_{k} \zeta_{k}(\omega) f_{k}(t)
$$

where $f_{k}: T \rightarrow \mathbb{C}$ are measurable functions such that

$$
\sum_{k}\left|f_{k}(t)\right|^{2}<\infty \quad \text { for all } t \in T
$$

and $\zeta_{k}=\xi_{k}+i \eta_{k}$ are independent standard complex Gaussian variables; i.e. $\zeta_{k} \sim \mathcal{N}_{\mathbb{C}}(0,1)$. The latter means that $\xi_{k}$ and $\eta_{k}$ are independent centered Gaussian (real) variables with variance $\frac{1}{2}$; then $\mathbb{E} \zeta_{k}=0$ and $\mathbb{E}\left|\zeta_{k}\right|^{2}=1$. We restrict ourselves to the case

$$
\sum_{k}\left|f_{k}(t)\right|^{2}=1 \quad \text { for all } t \in T \text {. }
$$

Then $w(t) \sim \mathcal{N}_{\mathbb{C}}(0,1)$ for all $t \in T$.

\footnotetext{
${ }^{6}$ In fact, it is the event $\sum\left|\zeta_{k}\right|^{2}=\infty$, of probability 1 .
} 
Now, a few words about the convergence of the series (2.1). Treating each term $\zeta_{k}(\omega) f_{k}(t)$ as an element of the space $\mathcal{L}^{2}=L^{2}((\Omega, P) \times(T, \mu))$, we have

$$
\left\|\zeta_{k} f_{k}\right\|_{\mathcal{L}^{2}}=\left\|\zeta_{k}\right\|_{L^{2}(\Omega, P)}\left\|f_{k}\right\|_{L^{2}(T, \mu)}=\left\|f_{k}\right\|_{L^{2}(T, \mu)}
$$

and the terms are pairwise orthogonal. Therefore, the series (2.1) converges in the space $^{7} \mathcal{L}^{2}$.

In what follows, we always assume that the sum of the series (2.1) is not the zero function in the space $\mathcal{L}^{2}$.

The correlation function $\rho: T \times T \rightarrow \mathbb{C}$ of the process $w(t)$ equals

$$
\rho(s, t) \stackrel{\text { def }}{=} \mathbb{E}\{w(s) \overline{w(t)}\}=\sum_{k} f_{k}(s) \overline{f_{k}(t)} .
$$

Clearly, $|\rho(s, t)| \leq 1$ and $\rho(t, t)=1$.

Consider a sequence of complex Gaussian processes $w_{n}$ with the correlation functions $\rho_{n}(s, t)$ and define a sequence of random variables

$$
Z_{n}=\int_{T} \varphi\left(\left|w_{n}(t)\right|\right) \Theta(t) d \mu(t)
$$

where $\varphi: \mathbb{R}_{+} \rightarrow \mathbb{R}$ is a measurable function such that

$$
\int_{0}^{\infty} \varphi^{2}(r) e^{-r^{2} / 2} r d r<\infty,
$$

and $\Theta: T \rightarrow \mathbb{R}$ is a measurable bounded function. We shall prove that under some natural assumptions on the off-diagonal decay of the correlation functions $\rho_{n}(s, t)$ when $n \rightarrow \infty$, the random variables $Z_{n}$ are asymptotically normal.

2.2 Theorem. Suppose that for each $\alpha \in \mathbb{N}$

$$
\liminf _{n \rightarrow \infty} \frac{\iint_{T^{2}}\left|\rho_{n}(s, t)\right|^{2 \alpha} \Theta(s) \Theta(t) d \mu(s) d \mu(t)}{\sup _{s \in T} \int_{T}\left|\rho_{n}(s, t)\right| d \mu(t)}>0,
$$

and that

$$
\lim _{n \rightarrow \infty} \sup _{s \in T} \int_{T}\left|\rho_{n}(s, t)\right| d \mu(t)=0 .
$$

${ }^{7}$ Moreover, for each $t \in T$, the series (2.1) converges in $L^{2}(\Omega, P)$ (for an obvious reason), and by the Kolmogorov-Khinchin theorem [15] Ch. 3] the series converges in $L^{2}(T, \mu)$ for almost all $\omega \in \Omega$. 
Then the distributions of the random variables

$$
\frac{Z_{n}-\mathbb{E} Z_{n}}{\sqrt{\operatorname{Var} Z_{n}}}
$$

converge weakly to $\mathcal{N}(0,1)$ for $n \rightarrow \infty$.

If $\varphi$ is an increasing function, then it suffices to assume that condition (2.3) holds only for $\alpha=1$.

\section{Remarks:}

(i) The function $|\rho(s, t)|^{2 \alpha}$ which appears in the integrand on the left-hand side of condition (2.3) is a positive definite function on $T \times T$ (see formula (2.6) below). Hence, for $\alpha \in \mathbb{N}$, the integral $\iint_{T^{2}}|\rho(s, t)|^{2 \alpha} \Theta(s) \Theta(t) d \mu(s) d \mu(t)$ is always non-negative.

(ii) The role of condition (2.4) is to guarantee that

$$
\lim _{n \rightarrow \infty} \operatorname{Var} Z_{n}=0
$$

In fact, under assumption (2.3), the sequences

$$
n \mapsto \operatorname{Var} Z_{n} \quad \text { and } \quad n \mapsto \int_{T}\left|\rho_{n}(s, t)\right| d \mu(t)
$$

have the same decay (see expression (2.7) below).

\subsection{Some preliminaries}

We shall deal with the space $L_{\mathbb{C}}^{2} \stackrel{\text { def }}{=} L^{2}\left(\mathbb{C}, \mathcal{N}_{\mathbb{C}}(0,1)\right)$ and its subspaces $\mathcal{P}_{m}$ which consist of the polynomials in $\zeta$ and $\bar{\zeta}$ of degrees $\alpha$ and $\beta$ correspondingly, $\alpha+\beta=m$. The space $L_{\mathbb{C}}^{2}$ has a polynomial basis

$$
\left\{\frac{1}{\sqrt{\alpha ! \beta !}}: \zeta^{\alpha} \bar{\zeta}^{\beta}:\right\}_{\alpha, \beta \in \mathbb{Z}_{+}}
$$

where : $\zeta^{\alpha} \bar{\zeta}^{\beta}$ : is the orthogonal projection of the polynomial $\zeta^{\alpha} \bar{\zeta}^{\beta}$ onto the subspace $H^{: m} \stackrel{\text { def }}{=} \mathcal{P}_{m} \ominus \mathcal{P}_{m-1}, m=\alpha+\beta$ [14, Example 3. 32]. ${ }^{8}$ Thus any square integrable function $\Phi$ of a random variable $\zeta \sim \mathcal{N}_{\mathbb{C}}(0,1)$ is of the form

$$
\Phi(\zeta)=\sum_{\alpha, \beta \in \mathbb{Z}_{+}} \frac{c_{\alpha \beta}}{\sqrt{\alpha ! \beta !}}: \zeta^{\alpha} \bar{\zeta}^{\beta}:, \quad\|\Phi\|^{2}=\sum_{\alpha, \beta}\left|c_{\alpha \beta}\right|^{2} .
$$

${ }^{8}$ The expression : $\zeta^{\alpha} \bar{\zeta}^{\beta}$ : is called the Wick product. 
In what follows, we shall deal only with the radial functions $\varphi(|\zeta|)$. In this case,

$$
\varphi(|\zeta|)=\sum_{\alpha=0}^{\infty} \frac{c_{2 \alpha}}{\alpha !}:|\zeta|^{2 \alpha}
$$

where $c_{2 \alpha} \in \mathbb{R}$ and $\sum_{\alpha} c_{2 \alpha}^{2}=\|\varphi\|^{2}$. Indeed, the group of rotations $\zeta \mapsto e^{i \theta} \zeta$ acts on the space $L_{\mathbb{C}}^{2}$ leaving invariant the subspaces $H^{: m}$ : If $m$ is odd, then $H^{:} m$ : contains no radial functions; if $m=2 \alpha$ is even, then the subspace of radial polynomials in $H^{: m:}$ is one-dimensional and is spanned by $:|\zeta|^{2 \alpha}:=$ $: \zeta^{\alpha} \bar{\zeta}^{\alpha}:$

In the proof of Theorem 2.2, we may assume without loss of generality that

$$
\int_{\mathbb{C}} \varphi(|\zeta|) e^{-|\zeta|^{2} / 2} d m(\zeta)=0
$$

that is, $\mathbb{E} Z_{n}=0$. Then expansion (2.5) starts with $\alpha=\alpha_{0} \geq 1, c_{2 \alpha_{0}} \neq 0$.

Since

$$
\mathbb{E}\left(\frac{1}{\alpha !}:|w(s)|^{2 \alpha}:\right)\left(\frac{1}{\beta !}:|w(t)|^{2 \beta}:\right)= \begin{cases}|\rho(s, t)|^{2 \alpha} & \text { if } \alpha=\beta \\ 0 & \text { otherwise }\end{cases}
$$

14. Theorem 3.9] (see also Example at the end of the next subsection), we have

$$
\mathbb{E}\left\{\varphi\left(\left|w_{n}(s)\right|\right) \varphi\left(\left|w_{n}(t)\right|\right)\right\}=\sum_{\alpha \geq \alpha_{0}} c_{2 \alpha}^{2}\left|\rho_{n}(s, t)\right|^{2 \alpha}
$$

and

$$
\begin{aligned}
\mathbb{E} Z_{n}^{2} & =\mathbb{E}\left(\int_{T} \varphi\left(\left|w_{n}(t)\right|\right) \Theta(t) d \mu(t)\right)^{2} \\
& =\iint_{T^{2}} \mathbb{E}\left\{\varphi\left(\left|w_{n}(s)\right|\right) \varphi\left(\left|w_{n}(t)\right|\right)\right\} \Theta(s) \Theta(t) d \mu(s) d \mu(t) \\
& =\sum_{\alpha \geq \alpha_{0}} c_{2 \alpha}^{2} \iint_{T^{2}}\left|\rho_{n}(s, t)\right|^{2 \alpha} \Theta(s) \Theta(t) d \mu(s) d \mu(t) .
\end{aligned}
$$

The proof of Theorem 2.2 uses the classical method of moments, though it cannot be applied directly to the random variables $Z_{n}$ since $\varphi$ (and therefore, $Z_{n}$ ) need not have more than two moments. First, we approximate $\varphi$ by the polynomials

$$
\varphi_{m}(|\zeta|)=\sum_{\alpha \leq m} \frac{c_{2 \alpha}}{\alpha !}:|\zeta|^{2 \alpha}: \quad m \geq \alpha_{0}
$$


and $Z_{n}$ by the random variables

$$
Z_{n, m}=\int_{T} \varphi_{m}\left(\left|w_{n}(t)\right|\right) \Theta(t) d \mu(t) .
$$

Then the moment method will be applied to $Z_{n, m}$.

Applying formula (2.7) to $Z_{n}-Z_{n, m}$ (that is, replacing the function $\varphi$ by $\left.\varphi-\varphi_{m}\right)$, we get

$$
\begin{aligned}
\mathbb{E}\left(Z_{n}-Z_{n, m}\right)^{2} & =\sum_{\alpha \geq m+1} c_{2 \alpha}^{2} \iint_{T^{2}}\left|\rho_{n}(s, t)\right|^{2 \alpha} \Theta(s) \Theta(t) d \mu(s) d \mu(t) \\
\leq\|\Theta\|_{L^{\infty}(T, \mu)}^{2} \sum_{\alpha \geq m+1} & c_{2 \alpha}^{2} \sup _{s \in T} \int_{T}\left|\rho_{n}(s, t)\right| d \mu(t) \\
& \stackrel{\underline{2.33}}{\leq} \frac{\|\Theta\|_{L^{\infty}(T, \mu)}^{2}}{\eta\left(\alpha_{0}\right)}\left\|\varphi-\varphi_{m}\right\|_{L_{\mathbb{C}}^{2}}^{2} \iint_{T^{2}}\left|\rho_{n}(s, t)\right|^{2 \alpha_{0}} \Theta(s) \Theta(t) d \mu(s) d \mu(t)
\end{aligned}
$$

where $\eta(\alpha)$ denotes the expression on the left-hand side of (2.3). Using again formula (2.7), we finally get

$$
\mathbb{E}\left(Z_{n}-Z_{n, m}\right)^{2} \leq \frac{\|\Theta\|_{L^{\infty}(T, \mu)}^{2}}{c_{2 \alpha_{0}}^{2} \eta\left(\alpha_{0}\right)}\left\|\varphi-\varphi_{m}\right\|_{L_{\mathbb{C}}^{2}}^{2} \mathbb{E} Z_{n}^{2}
$$

Hence

$$
\lim _{m \rightarrow \infty} \sup _{n} \mathbb{E}\left(\frac{Z_{n}}{\sqrt{\operatorname{Var} Z_{n}}}-\frac{Z_{n, m}}{\sqrt{\operatorname{Var} Z_{n, m}}}\right)^{2}=0
$$

and if for each fixed $m \geq \alpha_{0}$ the random variables $Z_{n, m} / \sqrt{\operatorname{Var} Z_{n, m}}$ converge in distribution to $\mathcal{N}(0,1)$ when $n \rightarrow \infty$, then the random variables $Z_{n} / \sqrt{\operatorname{Var} Z_{n}}$ have the same property.

From now on, we always assume that $\varphi$ is a polynomial (and hence $Z_{n}$ has the moments of all orders).

\subsection{More preliminaries (the diagram formula)}

The next step is to evaluate the moments $\mathbb{E} Z_{n}^{p}, p \in \mathbb{N}$, and to compare them with $\left(\mathbb{E} Z_{n}^{2}\right)^{p / 2}$ times the moments of the standard normal distribution.

We have

$$
\mathbb{E} Z_{n}^{p}=\int \cdots \int_{T^{p}} \mathbb{E}\left\{\prod_{j=1}^{p} \varphi\left(\left|w_{n}\left(t_{j}\right)\right|\right)\right\} \prod_{j=1}^{p} \Theta\left(t_{j}\right) d \mu\left(t_{j}\right)
$$

$\stackrel{\text { [2.5) }}{=} \sum_{\alpha_{1}, \ldots, \alpha_{p} \geq \alpha_{0}} \frac{c_{2 \alpha_{1}} \ldots c_{2 \alpha_{p}}}{\alpha_{1} ! \ldots \alpha_{p} !} \int \cdots \int_{T^{p}} \mathbb{E}\left\{\prod_{j=1}^{p}:\left|w_{n}\left(t_{j}\right)\right|^{2 \alpha_{j}}:\right\} \prod_{j=1}^{p} \Theta\left(t_{j}\right) d \mu\left(t_{j}\right)$. 
We compute the integrand $\mathbb{E}\left\{\prod_{j=1}^{p}:\left|w_{n}\left(t_{j}\right)\right|^{2 \alpha_{j}}:\right\}$ using the diagram technique [14, Chapter 3].

Fix the exponents $\alpha_{1}, \ldots, \alpha_{p}$. A diagram $\gamma$ is a graph with $2\left(\alpha_{1}+\cdots+\alpha_{p}\right)$ vertices labeled by the indices $1, \overline{1}, 2, \overline{2}, \ldots, p, \bar{p}\left(\alpha_{j}\right.$ vertices are labeled by $j$ and other $\alpha_{j}$ vertices are labeled by $\bar{j}$ ), and each vertex has degree one (i.e., the edges have no common end points). The edges may connect only the vertices labeled by $i$ and $\bar{j}$ with $i \neq j$. The set of all such graphs is denoted by $\Gamma\left(\alpha_{1}, \ldots, \alpha_{p}\right)$. For some choices of $\alpha_{1}, \ldots, \alpha_{p}, \Gamma\left(\alpha_{1}, \ldots, \alpha_{p}\right)$ may be the empty set, for example, $\Gamma\left(\alpha_{1}, \alpha_{2}\right)=\emptyset$ iff $\alpha_{1} \neq \alpha_{2}$, and $\Gamma\left(\alpha_{1}, \alpha_{2}, \alpha_{3}\right)=\emptyset$ if $\alpha_{1}>\alpha_{2}+\alpha_{3}$. The value of the diagram $\gamma$ equals

$$
V_{\gamma}\left(t_{1}, \ldots, t_{p}\right) \stackrel{\text { def }}{=} \prod_{(i, \bar{j}) \in e(\gamma)} \rho\left(t_{i}, t_{j}\right)
$$

where the product is taken over all edges of $\gamma$. In this notation, the diagram formula [14, Theorem 3.12] $\operatorname{reads}^{9}$

$$
\mathbb{E}\left\{\prod_{j=1}^{p}:\left|w_{n}\left(t_{j}\right)\right|^{2 \alpha_{j}}:\right\}=\sum_{\gamma \in \Gamma\left(\alpha_{1}, \ldots, \alpha_{p}\right)} V_{\gamma}\left(t_{1}, \ldots, t_{p}\right)
$$

(as usual, summation over the empty set means that the sum has zero value).

2.10 Example. Consider the simplest case $p=2$. If $\alpha_{1} \neq \alpha_{2}$, then $\Gamma\left(\alpha_{1}, \alpha_{2}\right)=\emptyset$, and $\mathbb{E}\left\{:\left|w\left(t_{1}\right)\right|^{2 \alpha_{1}}::\left|w\left(t_{2}\right)\right|^{2 \alpha_{2}}:\right\}=0$. Now, suppose $\alpha_{1}=$ $\alpha_{2}=\alpha$. Then we can glue together the vertices labeled by the same indices. We get a graph with four vertices and two edges of multiplicity $\alpha$ :

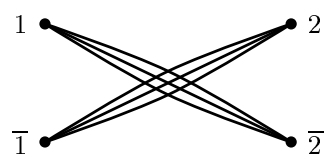

The edges connecting 1 and $\overline{2}$ contribute the factor $\rho\left(t_{1}, t_{2}\right)^{\alpha}$, the other $\alpha$ edges contribute the factor $\rho\left(t_{2}, t_{1}\right)^{\alpha}={\overline{\rho\left(t_{1}, t_{2}\right)}}^{\alpha}$. Thus the value of the diagram is $\left|\rho\left(t_{1}, t_{2}\right)\right|^{2 \alpha}$, and $\mathbb{E}\left\{:\left|w\left(t_{1}\right)\right|^{2 \alpha}::\left|w\left(t_{2}\right)\right|^{2 \alpha}:\right\}=\sharp \Gamma(\alpha, \alpha) \cdot\left|\rho\left(t_{1}, t_{2}\right)\right|^{2 \alpha}$. It remains to find the total number of diagrams in $\Gamma(\alpha, \alpha)$.

All the diagrams in $\Gamma(\alpha, \alpha)$ can be obtained from the fixed one by permutation of $\alpha$ vertices labeled by 1 , and by another independent permutation of $\alpha$ vertices labeled by 2 . Therefore, $\sharp \Gamma(\alpha, \alpha)=(\alpha !)^{2}$, and we recover formula (2.6).

\footnotetext{
${ }^{9}$ The diagrams we consider do not contain edges which join the vertices labeled by $i$ and $j$ (and by $\bar{i}$ and $\bar{j}$ ) since $\mathbb{E}\left\{w\left(t_{i}\right) w\left(t_{j}\right)\right\}=\mathbb{E}\left\{\overline{w\left(t_{i}\right)} \overline{w\left(t_{j}\right)}\right\}=0$.
} 


\subsection{The main argument}

For $p$ even, there are diagrams with a simple structure whose total contribution to $\mathbb{E} Z_{n}^{p}$ equals $\left(\mathbb{E} Z_{n}^{2}\right)^{p / 2} \mathbb{E} \xi^{p}$, where $\xi \sim \mathcal{N}(0,1)$. Contribution of the other diagrams is negligible.

2.12 Definition. A diagram $\gamma$ is called regular if the set $\{1,2, \ldots, p\}$ is split into $q=p / 2$ pairs and there are no edges between the vertices $i$ and $\bar{j}$ if $i$ and $j$ belong to different pairs. Otherwise, the diagram is called irregular.

In other words, the diagram is regular if after glueing together the vertices labeled by the same index it becomes a disjoint union of $q=p / 2$ "elementary diagrams" drawn on (2.11) (having, generally speaking, different multiplicities of the edges).

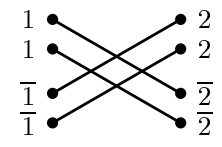

regular diagram

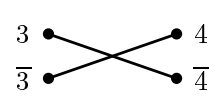

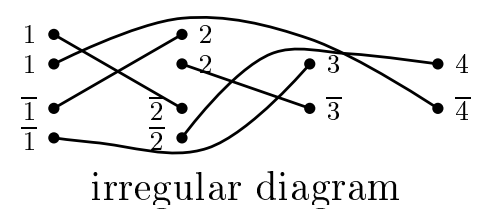

Suppose $p$ is even and the diagram $\gamma$ is regular. For each $j$, we glue together all the vertices labeled by $j$ and $\bar{j}$, obtaining a reduced diagram $\gamma^{*}$; i.e., a graph with $p$ vertices and multiple edges which is split into $q=p / 2$ connected components.

For example, if $\gamma$ is related to the partition

$$
\{1,2, \ldots, p\}=\{1,2\} \cup\{3,4\} \cup \cdots \cup\{p-1, p\},
$$

then the reduced diagram $\gamma^{*}$ is
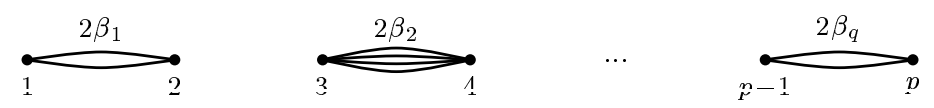

where $2 \beta_{k}=2 \alpha_{2 k-1}=2 \alpha_{2 k}$ is the multiplicity of the edge which connects the vertices $2 k-1$ and $2 k$. The $k$-th component of the reduced diagram $\gamma^{*}$ contributes by the factor $\left|\rho\left(t_{2 k-1}, t_{2 k}\right)\right|^{2 \beta_{k}}$, so that the value of diagram $\gamma$ is $\prod_{k=1}^{q}\left|\rho\left(t_{2 k-1}, t_{2 k}\right)\right|^{2 \beta_{k}}$. Integrating this over $T^{p}$ against the measure $\prod_{l=1}^{p} \Theta\left(t_{l}\right) d \mu\left(t_{l}\right)$, we get

$$
\prod_{k=1}^{q} \iint_{T^{2}}|\rho(s, t)|^{2 \beta_{k}} \Theta(s) \Theta(t) d \mu(s) d \mu(t) .
$$

Now we need to do some counting. Each reduced diagram $\gamma^{*}$ is defined by the choice of the partition, like (2.13), and the multiplicities $\beta_{1}, \ldots, \beta_{q}$ of the 
edges. Since the glueing procedure is not one-to-one, each reduced diagram has a "multiplicity" (that is, the number of regular diagrams which give the same reduced diagram $\gamma^{*}$ after glueing) depending on $\beta_{1}, \ldots, \beta_{q}$ which also should be taken into account.

Notice that if we started with a different partition of the set $\{1,2, \ldots, p\}$ into disjoint pairs, then anyway we would finish with the same answer (2.14). Therefore, we must multiply the expression (2.14) by the number of partitions of the set $\{1,2, \ldots, p\}$ into disjoint pairs. The number of such partitions equals $\mathbb{E} \xi^{p}$ where $\xi \sim \mathcal{N}(0,1)$ [14, Remark 1.29].

Next, let us count the "multiplicity" of the reduced diagram. If we fix a regular diagram $\gamma$, then all other regular diagrams having the same reduced diagram $\gamma^{*}$ can be obtained from $\gamma$ by $p$ independent permutations: we can permute $\alpha_{1}$ vertices labeled by 1 , then $\alpha_{2}$ vertices labeled by 2 and so on. Since these permutations are independent, the total multiplicity of the reduced diagram equals

$$
\alpha_{1} ! \alpha_{2} ! \ldots \alpha_{p} !=\left(\beta_{1} !\right)^{2}\left(\beta_{2} !\right)^{2} \ldots\left(\beta_{q} !\right)^{2} \text {, }
$$

and we need to put this factor before the product (2.14) when we summate over reduced diagrams; i.e. over all possible choices of the numbers $\beta_{1}, \ldots, \beta_{q} \geq \alpha_{0}$.

Combining all pieces, we get

$$
\begin{gathered}
\langle\text { regular }\rangle=\sum_{\beta_{1}, \ldots, \beta_{q} \geq \alpha_{0}} \frac{c_{2 \beta_{1}}^{2} \ldots c_{2 \beta_{q}}^{2}}{\left(\beta_{1} !\right)^{2} \ldots\left(\beta_{q} !\right)^{2}}\left(\beta_{1} !\right)^{2} \ldots\left(\beta_{q} !\right)^{2} \mathbb{E} \xi^{p}\langle\text { product (2.14) }\rangle \\
=\left(\mathbb{E} \xi^{p}\right)\left(\sum_{\beta \geq \alpha_{0}} c_{2 \beta}^{2} \iint_{T^{2}}|\rho(s, t)|^{2 \beta} \Theta(s) \Theta(t) d \mu(s) d \mu(t)\right)^{q} \stackrel{\sqrt{2.7}}{=}\left(\mathbb{E} \xi^{p}\right)\left(\mathbb{E} Z_{n}^{2}\right)^{p / 2} .
\end{gathered}
$$

Therefore,

$$
\mathbb{E} Z_{n}^{p}=\left(\mathbb{E} \xi^{p}\right)\left(\mathbb{E} Z_{n}^{2}\right)^{p / 2}+\langle\text { irregular }\rangle,
$$

and we need to show that the contribution of irregular diagrams is negligible with respect to the main term $\left(\mathbb{E} Z_{n}^{2}\right)^{p / 2}$ when $n \rightarrow \infty$.

Since $\varphi$ is a polynomial, there are only finitely many irregular diagrams which enter expression (2.8) for $\mathbb{E} Z_{n}^{p}$ after plugging in the diagram formula (2.9). We shall show that if the diagram $\gamma$ is irregular, then

$$
\int \ldots \int_{T^{p}}\left|V_{\gamma}\left(t_{1}, t_{2}, \ldots, t_{p}\right)\right| d \mu\left(t_{1}\right) \ldots d \mu\left(t_{p}\right)=o\left(\left(\sup _{s \in T} \int_{T}\left|\rho_{n}(s, t)\right| d \mu(t)\right)^{p / 2}\right),
$$


for $n \rightarrow \infty$. Then due to assumption (2.3) (and expression (2.7) for $\mathbb{E} Z_{n}^{2}$ )

$$
\int \ldots \int_{T^{p}}\left|V_{\gamma}\left(t_{1}, t_{2}, \ldots, t_{p}\right)\right| d \mu\left(t_{1}\right) \ldots d \mu\left(t_{p}\right)=o\left(\left(\mathbb{E} Z_{n}^{2}\right)^{p / 2}\right),
$$

and

$$
\langle\text { irregular }\rangle=o\left(\left(\mathbb{E} Z_{n}^{2}\right)^{p / 2}\right), \quad n \rightarrow \infty,
$$

which finishes the proof of Theorem 1.2 in the case of general radial functions up to the proof of (2.16).

Proof of (2.16) : First, we make another reduction of the diagram and define a $p$-vertex graph with simple edges which couple the vertices $i$ and $j$ if and only if at least one of the pairs $(i, \bar{j})$ or $(\bar{j}, i)$ was coupled in the original diagrams (without taking into account the multiplicities of the original coupling). We denote the reduced diagram by $\gamma^{* *}$. For example:

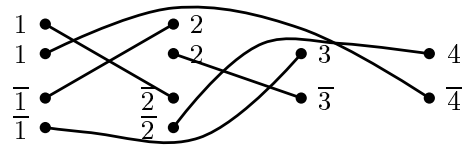

a diagram, $\gamma$

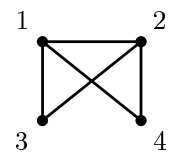

its reduction, $\gamma^{* *}$

Then

$$
\left|V_{\gamma}\left(t_{1}, \ldots, t_{p}\right)\right| \leq \prod_{(i, j) \in e\left(\gamma^{* *}\right)}\left|\rho\left(t_{i}, t_{j}\right)\right|
$$

where the product is taken over all edges of $\gamma^{* *}$. We have to estimate from above the integral of $\left|V_{\gamma}\right|$ over $T^{p}$. Replacing $\left|V_{\gamma}\right|$ by its upper bound (2.17), we obtain the integral which factorizes into the product of integrals described by connected components of the diagram $\gamma^{* *}$.

Let us start with one $m$-vertex component of the diagram $\gamma^{* *}$. The component can be a complicated graph - anyway, we can always turn this graph into a tree with $m$ vertices by deleting some edges (this procedure only increases the integral we are estimating). Having a tree, we choose a vertex belonging to only one edge and integrate it out, which gives the factor $\left(\sup _{s \in T} \int_{T}\left|\rho_{n}(s, t)\right| d \mu(t)\right)$ and the rest of the tree which is a new tree with $m-1$ vertices. By induction, any $m$-vertex tree describes the integral which does not exceed

$$
\left(\sup _{s \in T} \int_{T}\left|\rho_{n}(s, t)\right| d \mu(t)\right)^{m-1} .
$$

Now, suppose the reduced diagram $\gamma^{* *}$ has $k$ connected components and the $i$-th component has $m_{i}$ vertices. $^{10}$ Then the right-hand side of (2.17)

\footnotetext{
${ }^{10}$ Observe that $m_{1}+\cdots+m_{k}=p$.
} 
integrated over $T^{p}$ does not exceed

$$
\left(\sup _{s \in T} \int_{T}\left|\rho_{n}(s, t)\right| d \mu(t)\right)^{\left(m_{1}-1\right)+\cdots+\left(m_{k}-1\right)}=\left(\sup _{s \in T} \int_{T}\left|\rho_{n}(s, t)\right| d \mu(t)\right)^{p-k} .
$$

Since the diagram $\gamma$ is irregular, $k<p / 2$ and we get (2.16).

\subsection{The last step}

It remains to explain why in the case when the function $\varphi$ is increasing, it suffices to assume that condition (2.3) holds only for $\alpha=1$.

In the proof given above, condition (2.3) was used only in the estimate

$$
\sup _{s \in T} \int_{T}\left|\rho_{n}(s, t)\right| d \mu(t) \leq \frac{\mathbb{E} Z_{n}^{2}}{c_{2 \alpha_{0}}^{2} \eta\left(\alpha_{0}\right)}
$$

where $\alpha_{0} \geq 1$ is the minimal positive integer such that $c_{2 \alpha_{0}} \neq 0$, and $\eta(\alpha)$ is the left-hand side of (2.3); i.e., we need condition (2.3) only with $\alpha=\alpha_{0}$. If the function $\varphi$ increases, then $\alpha_{0}=1$. Indeed, it is easy to find that $:|\zeta|^{2}:=|\zeta|^{2}-1$. Then

$c_{2}=\int_{0}^{\infty} \varphi(r)\left(r^{2}-1\right) r e^{-r^{2} / 2} d r=\int_{0}^{\infty}(\varphi(r)-\varphi(1))\left(r^{2}-1\right) r e^{-r^{2} / 2} d r>0$, concluding the proof of Theorem 1.2.

\section{Asymptotic normality for chaotic analytic zero points}

\subsection{From chaotic analytic zeroes to non-linear func- tionals of Gaussian processes}

Recall the random objects defined in the Introduction: the Gaussian analytic functions $\psi_{L}(z)$ (see (0.1), (0.2) and (0.3)), the random measures $n_{\psi_{L}}$ counting their zeroes, and the linear statistics $Z_{L}(h)$. Since

$$
2 \pi d n_{\psi}=\Delta \log |\psi| d m=\Delta^{*} \log |\psi| d m^{*}
$$

where $d m^{*}$ and $\Delta^{*}$ are the invariant measure and the invariant Laplacian on $\mathcal{M}$, we have

$$
Z_{L}(h)=\frac{1}{2 \pi} \int_{\mathcal{M}} \log \left|\psi_{L}(z)\right|\left(\Delta^{*} h\right)(z) d m^{*}(z)
$$


obtaining in each of the three cases a family of non-linear functionals of the complex-valued Gaussian process $\psi_{L}$ defined on $\mathcal{M}$. We normalize the process $\psi$ putting

$$
w_{L}(z)=\frac{\psi_{L}(z)}{\sqrt{\mathbb{E}\left|\psi_{L}(z)\right|^{2}}}= \begin{cases}\psi_{L}(z)\left(1+|z|^{2}\right)^{-L / 2} & (\text { elliptic) } \\ \psi_{L}(z) e^{-L|z|^{2} / 2} & \text { (flat) } \\ \psi_{L}(z)\left(1-|z|^{2}\right)^{L / 2} & \text { (hyperbolic) } .\end{cases}
$$

In the flat and hyperbolic cases we use a large real parameter instead of the large integer parameter used in Section 2. Note that the (non-Gaussian) random process $\log |w(z)|$ is stationary (that is, its distribution is invariant with respect to the corresponding group of isometries), though the Gaussian process $w(z)$ is not (because of the phase multipliers in (0.4)). In all three cases, $w(z) \sim \mathcal{N}_{\mathbb{C}}(0,1)$ for each $z$. We obtain

$$
Z_{L}(h)-\mathbb{E} Z_{L}(h)=\frac{1}{2 \pi} \int_{\mathbb{M}} \log \left|w_{L}\right| \Delta^{*} h d m^{*} .
$$

Indeed, the left-hand side has zero expectations since $\mathbb{E} \log \left|w_{L}(z)\right|=$ const, and the invariant Laplacians of the test functions are orthogonal to the constants. It remains to check conditions (2.3) (with $\alpha=1$ ) and (2.4) of Theorem 2.2. and to compute the asymptotics of the variance of the random variables $Z_{L}(h)$.

\subsection{Checking conditions (2.3) and (2.4)}

Let

$$
\rho_{L}\left(z_{1}, z_{2}\right)=\mathbb{E}\left(w_{L}\left(z_{1}\right) \overline{w_{L}\left(z_{2}\right)}\right)=\frac{\mathbb{E}\left(\psi_{L}\left(z_{1}\right) \overline{\psi_{L}\left(z_{2}\right)}\right)}{\sqrt{\mathbb{E}\left|\psi_{L}\left(z_{1}\right)\right|^{2} \cdot \mathbb{E}\left|\psi_{L}\left(z_{2}\right)\right|^{2}}} .
$$

The function of two variables, $\left|\rho_{L}\left(z_{1}, z_{2}\right)\right|$, reduces to a function of one variable, $\left|\rho_{L}(z, 0)\right|$, by $G$-invariance, $\left|\rho_{L}\left(z_{1}, z_{2}\right)\right|=\left|\rho_{L}\left(g\left(z_{1}\right), g\left(z_{2}\right)\right)\right|$. Explicit formulas for $\rho_{L}(z, 0)=\left|\rho_{L}(z, 0)\right|$ follow from (0.1), (0.2), (0.3) and (3.1); namely,

$$
\begin{array}{llrl}
\rho_{L}(z, 0) & =\left(1+|z|^{2}\right)^{-L / 2} & & \text { (elliptic), } \\
\rho_{L}(z, 0) & =\exp \left(-\frac{1}{2} L|z|^{2}\right) & & \text { (flat), } \\
\rho_{L}(z, 0) & =\left(1-|z|^{2}\right)^{L / 2} & & \text { (hyperbolic). }
\end{array}
$$

A straightforward inspection shows that in all three cases the functions $\frac{L}{2 \pi} \rho_{L}(z, 0)$ converge weakly to the point mass at the origin:

$$
\lim _{L \rightarrow \infty} \frac{L}{2 \pi} \int_{\mathcal{M}} \rho_{L}(z, 0) \Theta(z) d m^{*}(z)=\Theta(0)
$$


for any continuous test-function $\Theta$ (as usual, with a compact support if $\mathcal{M}$ is non-compact).

By $G$-invariance, for any $\beta>0$, any continuous test-function $\Theta$ and any $z_{2} \in \mathcal{M}$,

$$
\lim _{L \rightarrow \infty} \frac{L \beta}{2 \pi} \int_{\mathcal{M}}\left|\rho_{L}\left(z_{1}, z_{2}\right)\right|^{\beta} \Theta\left(z_{1}\right) d m^{*}\left(z_{1}\right)=\Theta\left(z_{2}\right)
$$

(recall that $\left|\rho_{L}\right|^{\beta}=\left|\rho_{\beta L}\right|$ ). At last, multiplying the both sides of this equation by $\Theta\left(z_{2}\right)$ and integrating by $z_{2}$, we get

$$
\lim _{L \rightarrow \infty} \frac{L \beta}{2 \pi} \iint_{\mathcal{M}^{2}}\left|\rho_{L}\left(z_{1}, z_{2}\right)\right|^{\beta} \Theta\left(z_{1}\right) \Theta\left(z_{2}\right) d m^{*}\left(z_{1}\right) d m^{*}\left(z_{2}\right)=\|\Theta\|_{L^{2}\left(m^{*}\right)}^{2} .
$$

Now, conditions (2.3) and (2.4) become evident: for $L \rightarrow \infty$,

$$
\sup _{z_{2} \in \mathcal{M}} \int_{\mathcal{M}}\left|\rho_{L}\left(z_{1}, z_{2}\right)\right| d m^{*}\left(z_{1}\right)=\int_{\mathcal{M}} \mid \rho_{L}\left(z, 0 \mid d m^{*}(z) \sim \frac{2 \pi}{L},\right.
$$

this gives us (2.4). To get (2.3), observe that the double integral in the numerator of (2.3) has the same decay

$$
\iint_{\mathcal{M}^{2}}\left|\rho_{L}\left(z_{1}, z_{2}\right)\right|^{2} \Theta\left(z_{1}\right) \Theta\left(z_{2}\right) d m^{*}\left(z_{1}\right) d m^{*}\left(z_{2}\right) \sim \frac{\pi}{L} \cdot\|\Theta\|_{L^{2}\left(m^{*}\right)}^{2} .
$$

\subsection{Asymptotics of the variance}

We shall use formula (2.7) for the variance of $Z(h)$,

$$
\mathbb{E} Z_{L}^{2}(h)=\frac{1}{4 \pi^{2}} \sum_{\alpha \geq 1} c_{2 \alpha}^{2} \iint_{\mathcal{M}^{2}}\left|\rho_{L}\left(z_{1}, z_{2}\right)\right|^{2 \alpha} \Delta^{*} h\left(z_{1}\right) \Delta^{*} h\left(z_{2}\right) d m^{*}\left(z_{1}\right) d m^{*}\left(z_{2}\right),
$$

where $c_{2 \alpha}$ are defined by the expansion

$$
\log |\zeta|=\sum_{\alpha=0}^{\infty} \frac{c_{2 \alpha}}{\alpha !}:|\zeta|^{2 \alpha}: \quad \text { for } \zeta \sim \mathcal{N}_{\mathbb{C}}(0,1)
$$

(cf. (2.5)). Denoting

$$
\kappa=\frac{1}{4 \pi} \sum_{\alpha \geq 1} \frac{c_{2 \alpha}^{2}}{\alpha},
$$

we reduce (0.6) to relation (3.2) with $\beta=2 \alpha$ :

$$
\mathbb{E} Z_{L}^{2}(h)=\frac{1+o(1)}{4 \pi^{2}} \sum_{\alpha \geq 1} c_{2 \alpha}^{2} \cdot \frac{\pi}{L \alpha} \cdot\left\|\Delta^{*} h\right\|_{L^{2}\left(m^{*}\right)}^{2}=\frac{\kappa+o(1)}{L} \cdot\left\|\Delta^{*} h\right\|_{L^{2}\left(m^{*}\right)}^{2} .
$$




\section{References}

[1] P. Bleher and D. Ridzal, SU $(1,1)$ random polynomials, J. Statist. Phys. 106 (2002), 147-171.

[2] P. Bleher, B. Shiffman and S. Zelditch, Poincaré-Lelong approach to universality and scaling of correlations between zeros, Comm. Math. Phys. 208 (2000), 771-785.

[3] P. Bleher, B. Shiffman and S. Zelditch, Universality and scaling of correlations between zeros on complex manifolds, Invent. Math. 142 (2000), 351-395.

[4] P. Bleher, B. Shiffman and S. Zelditch, Universality and scaling of zeros on symplectic manifolds, Random matrix models and their applications, 31-69, Math. Sci. Res. Inst. Publ., 40, Cambridge Univ. Press, Cambridge, 2001.

[5] E. Bogomolny, O. Bohigas, And P. Lebouef, Distribution of roots of random polynomials, Phys. Rev. Letters 68 (1992), 2726-2729.

[6] E. Bogomolny, O. Bohigas, And P. Lebouef, Quantum chaotic dynamics and random polynomials, J. Statist. Phys. 85 (1995), 639-679.

[7] P. Breuer And P. Major, Central limit theorems for nonlinear functionals of Gaussian fields, J. Multivariate Anal. 13 (1983), 425-441.

[8] E. Calabi, Isometric imbedding of complex manifolds, Ann. of Math. (2) 58 (1953), 1-23.

[9] A. Edelman and E. Kostlan, How many zeros of a random polynomial are real? Bull. Amer. Math. Soc. 32 (1995), 1-37.

[10] P. J. Forrester And G. Honner, Exact statistical properties of complex random polynomials, J. Phys. A: Math. Gen. 32 (1999), 29612981.

[11] M. Gromov, Convex sets and Kähler manifolds, Advances in Differential Geometry and Topology (F. Tricerri, ed.), 1-38. World Sci. Publishing, Teaneck, NJ, 1990.

[12] J. H. Hannay, Chaotic analytic zero points: exact statistics for those of a random spin state, J. Phys. A: Math. Gen. 29 (1996), L101-L105. 
[13] J. H. Hannay, The chaotic analytic function, J. Phys. A: Math. Gen. 31 (1998), L755-L761.

[14] S. Janson, Gaussian Hilbert Spaces, Cambridge Univ. Press, 1997.

[15] J.-P. Kahane, Some Random Series of Functions, Cambridge Univ. Press, 1985.

[16] E. Kostlan, On distribution of roots of random polynomials, From Topology to Computation: Proceedings of the Smalefest (M. W. Hirsch, J. E. Marsden, and M. Shub, eds), 419-431. Springer-Verlag, New York, 1993.

[17] P. Leboeuf, Random analytic chaotic eigenstates, J. Statist. Phys. 95 (1999), 651-664.

[18] N. B. Maslova, The variance of the number of real roots of random polynomials, Teor. Verojatnost. i Primenen. 19 (1974), 36-51. (Russian)

[19] N. B. Maslova, The distribution of the number of real roots of random polynomials, Teor. Verojatnost. i Primenen. 19 (1974), 488-500. (Russian)

[20] A. M. Perelomov, Generalized Coherent States and Their Applications, Springer, 1986.

[21] Yu. Peres and B. Virág, Zeros of the i.i.d. Gaussian power series and a conformally invariant determinantal process, arXiv: math.PR/0310297.

[22] B. Shiffman and S. Zelditch, Distribution of zeros of random and quantum chaotic sections of positive line bundles, Commun. Math. Phys. 200 (1999), 661-684.

[23] M. Shub And S. Smale, Complexity of Bezout's Theorem. I: Geometric Aspects; II: Volumes and Probabilities; III: Condition Number and Packing; IV: Probability of Success; Extensions; V: Polynomial Time, The Collected Papers of Stephen Smale, Vol. 3 (F. Cucker and R. Wong eds), 1359-1476. World Scientific, Singapore, 2000.

[24] M. Sodin, Zeros of Gaussian analytic functions, Math. Res. Lett. 7 (2000), 371-381.

[25] M. Sodin And B. Tsirelson, Random complex zeroes, II. Perturbed lattice. arXiv:math. CV/0309449. 
[26] A. Soshnikov, Determinantal random point fields, Russian Math. Surveys 55 (2000), 923-975.

[27] M. Spera, G. Valli, Remarks on Calabi's diastasis function and coherent states, Quart. J. Math. Oxford (2) 44, 497-512 (1993).

[28] S. ZELDitch, From random polynomials to symplectic geometry, XIIIth International Congress on Mathematical Physics (London, 2000), 367376, Int. Press, Boston, MA, 2001.

MikHail Sodin

School of Mathematics

Tel Aviv University

Tel Aviv 69978, IsRAel

sodin@tau.ac.il
BORIS TSIRELSON

SchoOl of Mathematics

Tel Aviv University

Tel Aviv 69978, IsRael

tsirel@tau.ac.il

www.tau.ac.il/ ${ }^{\text {tsirel/ }}$ 